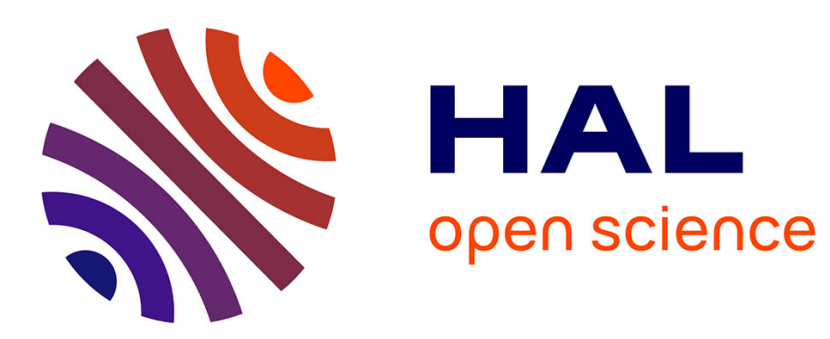

\title{
Détection de Pseudomonas phaseolicola (Burkh.) Dowson par la technique ELISA
}

\author{
Marie-Renée M.-R. Barzic, André Trigalet
}

\section{To cite this version:}

Marie-Renée M.-R. Barzic, André Trigalet. Détection de Pseudomonas phaseolicola (Burkh.) Dowson par la technique ELISA. Agronomie, 1982, 2 (4), pp.389-398. hal-02725790

\section{HAL Id: hal-02725790 \\ https://hal.inrae.fr/hal-02725790}

Submitted on 2 Jun 2020

HAL is a multi-disciplinary open access archive for the deposit and dissemination of scientific research documents, whether they are published or not. The documents may come from teaching and research institutions in France or abroad, or from public or private research centers.
L'archive ouverte pluridisciplinaire HAL, est destinée au dépôt et à la diffusion de documents scientifiques de niveau recherche, publiés ou non, émanant des établissements d'enseignement et de recherche français ou étrangers, des laboratoires publics ou privés. 


\section{Détection de Pseudomonas phaseolicola (Burkh.) Dowson par la technique ELISA}

Marie-Renée BARZIC \& André TRIGALET

I.N.R.A., Station de Pathologie végétale et Phytobactériologie, Beaucouzé, F 49000 Angers.

\section{RÉSUMÉ}

\section{ELISA,}

Détection,

Pseudomonas phaseolicola,

Phaseolus vulgaris,

Métabolites,

Sensibilité.
La technique ELISA a été appliquée à la détection de Pseudomonas phaseolicola dans des graines et des feuilles contaminées de haricot.

Le test ELISA a été réalisé selon la méthode sandwich d'après CLARK \& ADAMS (1977), modifiée par l'emploi de tampon substrat: Tris- $\mathrm{HCl}, \mathrm{NaCl}$ avec la phosphatase alcaline d'Escherichia coli.

Les immunoglobulines ont été purifiées par chromatographie d'affinité avec soit la protéine $A$, soit la bactérie entière Ps. phaseolicola comme ligand (fig. 1).

Des études préalables ont montré que la technique ElISA donnait des résultats positifs avec les suspensions bactériennes et les filtrats correspondants après passage sur membrane de $0,22 \mu \mathrm{m}$.

Dans nos conditions expérimentales, il ressort que :

- la limite de sensibilité de cette technique correspond à une suspension bactérienne de $10^{4}$ bactéries $/ \mathrm{ml}$ en $4 \mathrm{~h}$ de réaction (tabl. 1).

- Les métabolites bactériens contenus dans les filtrats donnent des réactions parallèles à celles obtenues avec les suspensions (tabl. 2 et 3 ).

- La concentration de métabolites solubles détectés est d'environ $10 \mathrm{ng}$ par $\mathrm{ml}$ par référence à la sérum albumine.

- La sensibilité de la technique Elisa ne peut être exprimée en terme de concentration bactérienne mais plutôt en terme de concentration d'antigènes bactériens, solubles ou non. Il serait d'un grand intérêt de tenter d'établir une corrélation entre une concentration bactérienne et la production de métabolites correspondant dans des conditions naturelles d'environnement.

Les résultats obtenus pour la détection de Ps. phaseolicola dans les graines et les feuilles de haricot contaminées par les techniques d'immunofluorescence ou ElisA sont identiques (tabl. 4 et 5).

L'extraction et la purification de quelques métabolites bactériens liés au pouvoir pathogène doivent conduire à lobtention d'antisérums spécifiques qui permettront des études qualitatives et quantitatives, par la technique EuISA, de la production et de la distribution de ces métabolites pathogènes in vivo.

La standardisation dans la purification des immunoglobulines et dans leur couplage avec une enzyme plus active est nécessaire pour permettre des comparaisons et pour améliorer la sensibilité de la technique ElisA.

\section{SUMMARY}

ELISA,

Detection,

Pscudomonas phaseolicola, Phaseolus vulgaris, Metabolites, Sensitivity.

\section{Detection of Pseudomonas phaseolicola (Burkh.) Dowson by the ELISA technique}

The ELISA technique has been applied to the detection of Pseudomonas phaseolicola in contaminated seed and leaves of bean.

ELISA tests were performed according to the sandwich method as described by CLARK \& ADAMS (1977) and slightly modified by using Tris- $\mathrm{HCl}$ saline buffer for the enzymatic phase with alkaline phosphatase from Escherichia coli.

Purified immunoglobulins were obtained by affinity chromatography using either protein $\mathrm{A}$, or whole bacterial Ps. phaseolicola as ligands (fig. 1).

Preliminary studies revealed that the ELISA technique was effective when applied to bacterial suspensions or to corresponding filtrates through $0.22 \mu \mathrm{m}$ membranes as well.

Under our experimental conditions we found :

i) that the lower limit of detection of the ELISA sandwich method was about $10^{4}$ bacteria per $\mathrm{ml}$ within four hours (table 1);

ii) that the bacterial metabolites in the filtrates led to heavy, rapid positive responses (tables 2 and 3 ); iii) that the detectable amount of soluble protein is about $10 \mathrm{ng}$ per $\mathrm{ml}$ by reference to serum albumin ; iv) that ELISA sensitivity could not be expressed in terms of bacterial concentration, but rather in terms of the concentration of bacterial antigens soluble or not. A question of major interest is to establish the possible correlation between a bacterial concentration and its related metabolite production under natural environmental conditions.

A good correlation was demonstrated between the immunofluorescence indirect method and ElisA in detecting Ps. phaseolicola in contaminated seeds and leaves of bean (tables 4 and 5).

The extraction and purification of some bacterial metabolites involved in pathogenicity, should lead to the production of specific antisera. These should be of great help in qualitative and quantitative studies by ELISA on the production and distribution of these pathogenic metabolites in vivo.

Standardization in purifying immunoglobulins and in conjugating them with more active enzymes is needed to allow valid comparisons and improve the sensitivity of ELISA. 


\section{INTRODUCTION}

L'utilisation des techniques sérologiques appliquées à la détection de bactéries phytopathogènes dans les milieux naturels contaminés n'a cessé de se développer depuis quelques années. Ces techniques sérologiques allient la spécificité de la réaction antigène-anticorps proprement dite à la sensibilité de résolution d'un système marqueur dont le plus communément utilisé a été, dès 1941, la molécule fluorescente (CoONs et al.), révélée en lumière violette (immunofluorescence). L'immunofluorescence est maintenant largement utilisée pour la sélection sanitaire vis-à-vis de nombreuses bactérioses au niveau des semences, boutures et tubercules (TrigalET et al., 1979). Les réactions immunoenzymatiques, quant à elles, ont pris leur essor depuis 1966 à la suite de travaux de NAKANE et al., d'une part, d'Avrameas \& URIEL, d'autre part. La possibilité de coupler des anticorps ou des antigènes à des enzymes était vérifiée. Depuis, l'application des réactions immunoenzymatiques s'est étendue à des domaines variés où interviennent non plus seulement la reconnaissance entre un antigène et l'anticorps correspondant mais, d'une manière générale, d'autres types d'interaction moléculaire telles que hormones-récepteurs, lectines-sucres (AVRAMEAS et al., 1976).

Le test immunoenzymatique ou ELISA (enzyme linked immunosorbent assay), considéré d'abord comme outil qualitatif pour la détection et la localisation d'antigènes (NAKANE et al., 1966; AVRAMEAS \& URIEL, 1966), a été appliqué à partir de 1971 par VAN VEEMEN \& SCHURRS, Avrameas \& Guilbert, Engvall et Perlmann à des fins quantitatives, ce qui explique l'intérêt que lui porte le domaine médical.

Dans le domaine de la pathologie végétale, la technique ELISA a d'abord été appliquée à la détection de virus. A ce propos, on doit rapporter les premiers travaux de VOLLER et al., en 1976, de ClARK \& ADAMS en 1977. En phytobactériologie, par contre, cette technique ne semble pas pour le moment avoir pris un grand essor. Seuls quelques travaux la signalent avec les modèles bactériens suivants: Corynebacterium sepedonicum (CAFLIN \& UYEMOTO, 1978), Pseudomonas phaseolicola (WEAVER \& GUTHRIE, 1978), Erwinia carotovora var. atroseptica (VRUGGING, 1978, CARON \& COPEMAN, 1981), Xanthomonas pelargonii (VRUGGING, 1978), Corynebacterium michiganense (STEVENS \& TSIANTOS, 1979). Une étude sur l'identification des souches de Rhizobium en culture pure et dans les nodules de lentilles a été présentée par BERGER et al., en 1979. CAMBRA \& LOPEZ (1978) préconisent cette technique non pas pour la détection du germe mais pour le titrage en anticorps des antisérums dirigés contre Agrobacterium radiobacter var. tumefaciens.

La sensibilité de la technique ELISA appliquée à la détection de bactéries phytopathogènes apparaît exprimée, dans ces différents travaux, en concentration de cellules détectées. Ainsi, toutes les études portant sur des cultures bactériennes pures en font mention et différentes valeurs sont proposées en fonction du germe étudié : $10^{3}$ bactéries/ml avec C. michiganense (STEVENS \& TSIANTOS, 1979); $10^{4}-10^{5}$ ou $10^{5}-10^{6} / \mathrm{ml}$ avec E. carotovora var. atroseptica suivant qu'il s'agit de cultures traitées à la chaleur ou non (CARon \& Copeman, 1981); $10^{6}$ à $10^{7} / \mathrm{ml}$ pour Ps. phaseolicola (Weaver \& Guthrie, 1978). Pour VRUGging (1978), la sensibilité de la méthode ElISA est qualitativement comparable à l'immunofluorescence. Par contre, avec les échantillons végétaux sous forme de broyats ou de liquides de macération, les résultats sont surtout qualitatifs.
Seuls STEvens \& Tsiantos (1979) donnent un seuil de sensibilité correspondant à $10^{6}$ bactéries/ml d'échantillon testé.

Notre étude a porté sur le modèle «maladie de la graisse à halo du haricot commun »due à Pseudomonas phaseolicola (Burkh.) Dowson. La détection de ce germe dans les semences a déjà fait l'objet de nombreuses publications. Différentes méthodes (isolement, phages, test germinatif, inoculation du liquide de macération des graines) ont été étudiées et apparaissent plus ou moins adaptées à la détection de la bactérie. La technique d'immunofluorescence (COLÉNO, 1968) est maintenant de pratique courante et permet la détection, dans les lots de semences de haricot, de $10^{3}$ bactéries/ml d'échantillon (TRIGALET et al., 1978). La technique ELISA, par ses possibilités d'automatisation et donc d'analyse de grandes séries d'échantillons, nous a paru intéressante comparativement aux méthodes déjà décrites. Nous avons cherché à préciser la sensibilité de détection de cette méthode appliquée à des cultures bactériennes pures et à du matériel végétal naturellement contaminé par Ps. phaseolicola.

\section{MATÉRIEL ET MÉTHODES}

\section{A. Réactifs}

\section{Immunoglobulines}

Les immunoglobulines sont obtenues à partir d'antisérums préparés chez le lapin et le poulet. La suspension antigénique est obtenue à partir d'une culture de la souche Ps. phaseolicola CNBP $(*), \mathrm{n}^{\circ} 1882$ sur milieu de Marten gélosé (bactopeptone 0,3 p. 100, bactotryptone 0,3 p. 100, extrait de levure 0,3 p. 100 , glucose 0,3 p. 100, agar 1,5 p. 100 , pH 7,0) pendant $24 \mathrm{~h}$ environ.

L'immunisation est pratiquée chez le lapin par injection par voie intraveineuse d'une suspension de cellules vivantes selon un protocole long déjà décrit (TRIGALET et al., 1978). Les prélèvements de sérums s'effectuent après le $4^{e}$ rappel.

Chez le poulet, l'immunisation se fait par voie souscutanée en 4 points du bréchet avec une suspension de cellules vivantes additionnée d'adjuvant complet de Freund : $0,5 \mathrm{ml}$ d'adjuvant pour $1,5 \mathrm{ml}$ de suspension bactérienne. Les injections sont faites à raison d'une piqûre par semaine pendant 3 semaines. Le prélèvement est fait 6 . après la dernière piqûre et après 3 rappels.

Les immunoglobulines sont extraites à partir de ces sérums par chromatographie d'affinité soit sur une colonne de Sépharose 4B - protéine A (Pharmacia Fine Chemicals), soit sur une colonne de Sépharose $4 \mathrm{~B}$ - cellules bactériennes: Ps. phaseolicola 1882 (BARZIC, 1980). Les immunoglobulines de poulet n'étant pas retenues sur la protéine $\mathrm{A}$, seul le $2^{\mathrm{e}}$ type de chromatographie d'affinité a été retenu pour leur extraction. Leur élution de la colonne d'affinité est obtenue avec un tampon $\mathrm{HCl}-$ glycine $0,1 \mathrm{M}$ $\mathrm{pH} 2,8$. Après neutralisation et concentration par ultrafiltration sur membrane Amicon PM 10, elles sont réparties en godets et stockées au congélateur. La concentration de la solution est déterminée d'après la valeur de la densité optique à $280 \mathrm{~nm}$.

(*) CNBP : Collection Nationale de Bactéries Phytopathogènes. I.N.R.A., Angers, 


\section{Echantillons}

\section{a) Bactéries}

Les bactéries suivantes ont été utilisées : Ps. phaseolicola souches CNBP $n^{\circ} 1882$, CNBP $n^{\circ} 1659$; Ps. syringae van Hall souches CNBP $n^{\circ} 1779, \mathrm{CNBP} \mathrm{n}^{\circ} 1769, \mathrm{CNBP} \mathrm{n}^{\circ} 1147$. Elles sont cultivées dans les conditions précédemment décrites. Les échantillons bactériens sont préparés selon 3 procédés différents.

- Les bactéries sont mises en suspension dans le liquide physiologique suivant: eau physiologique contenant du tween 20 et de l'azide de sodium aux concentrations finales respectives de 0,05 p. $100(\mathrm{v} / \mathrm{v})$ et 0,2 p. $100(\mathrm{p} / \mathrm{v}), \mathrm{pH} 7,4$. La suspension bactérienne (SB) est ajustée à 0,25 de densité optique à $660 \mathrm{~nm}$, ce qui correspond à une concentration de $3 \cdot 10^{8}$ bactéries $/ \mathrm{ml}$ d'après une courbe de référence établie par la méthode comptage par dilution sur milieu de Marten.

- La suspension bactérienne (SB) précédemment décrite est centrifugée $10 \mathrm{mn}$ à $4500 \mathrm{~g}$. Le surnageant de centrifugation est filtré sur membrane Millipore $0,22 \mu \mathrm{m}$ (FB).

- Le culot bactérien (C) obtenu par centrifugation de suspension bactérienne (SB), dont la concentration initiale est comprise entre $10^{9}$ et $10^{10}$ bactéries $/ \mathrm{ml}$, est remis en suspension dans le liquide physiologique. Un volume suffisant pour la réalisation du test ELISA est conservé à $4{ }^{\circ} \mathrm{C}$. La suspension bactérienne est centrifugée à nouveau $10 \mathrm{mn}$ à $4500 \mathrm{~g}$ et traitée comme précédemment. Ces opérations sont répétées plusieurs fois $(\mathrm{Ci}$ et $\mathrm{Si})$. Les surnageants de centrifugation ( $\mathrm{Si}$ ) sont filtrés sur $0,22 \mu \mathrm{m}$.

\section{b) Végétaux}

Les échantillons végétaux proviennent $\mathrm{du}$ haricot commun Phaseolus vulgaris L. var. «Coco nain blanc précoce », «Vernel », « Hanfa », «Amilcar », « Calvy », "Opal ». Ils sont constitués soit par des liquides de macération dans de l'eau stérile $16 \mathrm{~h}$ à $4{ }^{\circ} \mathrm{C}$ de semences $(200 \mathrm{~g}$ de graines pour $200 \mathrm{ml}$ d'eau stérile) saines ou naturellement contaminées par Ps. phaseolicola, soit par des broyats de feuilles saines ou artificiellement contaminées par pulvérisation par les souches citées précédemment. Les broyages de feuilles sont réalisés dans le liquide physiologique auquel sont ajoutés du bisulfite de sodium à saturation et du diéthyldithiocarbamate de sodium aux concentrations respectives de 1 p. $100(\mathrm{v} / \mathrm{v})$ et de 0,2 p. $100(\mathrm{p} / \mathrm{v})$. Les échantillons sont utilisés sous forme d'extrait brut (EB) ou après filtration sur membrane de $0,22 \mu \mathrm{m}$ (EF).

\section{B. Couplage des immunoglobulines avec la phosphatase}

Les immunoglobulines sont couplées à la phosphatase alcaline type III R (Sigma EC $n^{\circ} 3.1-3.1$ ) par l'intermédiaire du glutaraldéhyde (méthode en un temps) selon le procédé décrit par AVRAMEAs et al., en 1978. Le couplage est conservé au congélateur, dilué au $1 / 2$ dans du glycérol.

\section{Conduite de la réaction immunoenzymatique}

La réalisation du test ELISA est faite suivant la méthode sandwich d'après les travaux de CLARK \& ADAMS (1976) ou suivant la méthode de double couche d'après SCHERRER \& BERNARD (1977). Les réactions sont réalisées soit en plaques de microtitration (COOKE M $129 \mathrm{~A}$ ), soit en cuves Gilford.

\section{Méthode sandwich}

Les immunoglobulines $(300 \mu \mathrm{l}$ à $10 \mu \mathrm{g} / \mathrm{ml})$ sont réparties dans les cupules. L'adsorption est faite $5 \mathrm{~h}$ à $37^{\circ} \mathrm{C}$ ou 1 nuit à $4^{\circ} \mathrm{C}$. Après 3 lavages avec le liquide physiologique, le dépôt de l'échantillon est réalisé à raison de $200 \mu \mathrm{l}$ par essai. L'incubation se fait soit 1 nuit à $28^{\circ} \mathrm{C}$, soit $2 \mathrm{~h}$ à $28^{\circ} \mathrm{C}$. Après élimination de l'échantillon et lavages comme précédemment décrits, $250 \mu \mathrm{l}$ du couplage anticorps-enzyme dans le liquide physiologique additionné d'albumine bovine à 0,4 p. $100(\mathrm{p} / \mathrm{v})($ Sigma-fraction $\mathrm{V})$ sont laissés $4 \mathrm{~h}$ à $37^{\circ} \mathrm{C}$. La révélation de la réaction est obtenue avec $0,8 \mathrm{mg} / \mathrm{ml}$ de p. nitrophényl phosphate dans un tampon Tris- $\mathrm{HCl} 0,05 \mathrm{M}$, $\mathrm{NaCl} 1 \mathrm{M}$ pH 8,0 (GAREN \& LevinthaL, 1960). La lecture s'opère à $405 \mathrm{~nm}$ soit au spectrophotomètre Beckman DB$G$ dans des microcuves, soit avec le lecteur automatique Osi. La dilution d'emploi du couplage ou titre a été préalablement déterminée. La dilution utilisée est celle qui donne une densité optique de la réaction colorimétrique égale à 2 en $1 \mathrm{~h}$ de réaction avec $10^{8}$ bactéries $/ \mathrm{ml}$ comme échantillon et un témoin de réaction de densité optique inférieure à 0,1 . Dans chaque série de test figurent des témoins de réaction (TR ou immunoglobulines-liquide physiologique - conjugué anticorps enzyme - substrat) et des témoins tampon substrat avec le substrat $(\mathrm{TS}+\mathrm{S})$. La densité optique corrigée d'une réaction représente la valeur de sa densité optique diminuée de la densité optique du témoin de réaction.

\section{Méthode de double couche}

Les lavages des cupules entre les différentes étapes de la réaction s'opèrent comme précédemment. Le déroulement de la réaction se fait en plusieurs temps: garnissage des parois du support par adsorption des anticorps de poulet $\left(300 \mu \mathrm{l}\right.$ à $10 \mu \mathrm{g} / \mathrm{ml}, 5 \mathrm{~h}$ à $\left.37^{\circ} \mathrm{C}\right)$, dépôt de l'échantillon bactérien $\left(220 \mu \mathrm{l}, 1\right.$ nuit à $\left.28^{\circ} \mathrm{C}\right)$ puis des immunoglobulines de lapin $\left(250 \mu \mathrm{l}, 3 \mathrm{~h}\right.$ à $\left.37^{\circ} \mathrm{C}\right)$. Ces immunoglobulines sont utilisées diluées au $1 / 10$, la dilution de $1 / 160$ représentant le titre obtenu par la technique d'immunofluorescence et enfin du conjugué anticorps de mouton anti-immunoglobulines de lapin couplées à la phosphatase alcaline (Institut Pasteur production) dilué au $1 / 500\left(3 \mathrm{~h}\right.$ à $\left.37^{\circ} \mathrm{C}\right)$ avant la révélation de l'activité enzymatique comme ci-dessus.

\section{Le test d'immunofluorescence}

Le test d'immunofluorescence est réalisé selon la méthode indirecte avec un anti-sérum $P$ s. phaseolicola 1882 dilué au 1/1000 ou absorbé par Pseudomonas syringae $\mathrm{n}^{\circ} 1147$ et Pseudomonas $\mathrm{n}^{\circ} 379$ (groupe V) (TRIGALET $e t$ al., 1978)

Les frottis d'échantillons végétaux sont préparés sur des lames porte-objets. Les parois des cuves ont également été examinées par immunofluorescence pour vérifier la présence de cellules bactériennes.

\section{RÉSULTATS}

\section{A. Détection de la suspension bactérienne}

Des essais ont été réalisés pour mettre en évidence la détection de bactéries en culture pure. Trois types d'immunoglobulines ont été utilisés pour le garnissage des parois des cupules. L'observation de la figure 1 met en évidence l'efficience, dans la réaction ELISA, des anticorps 


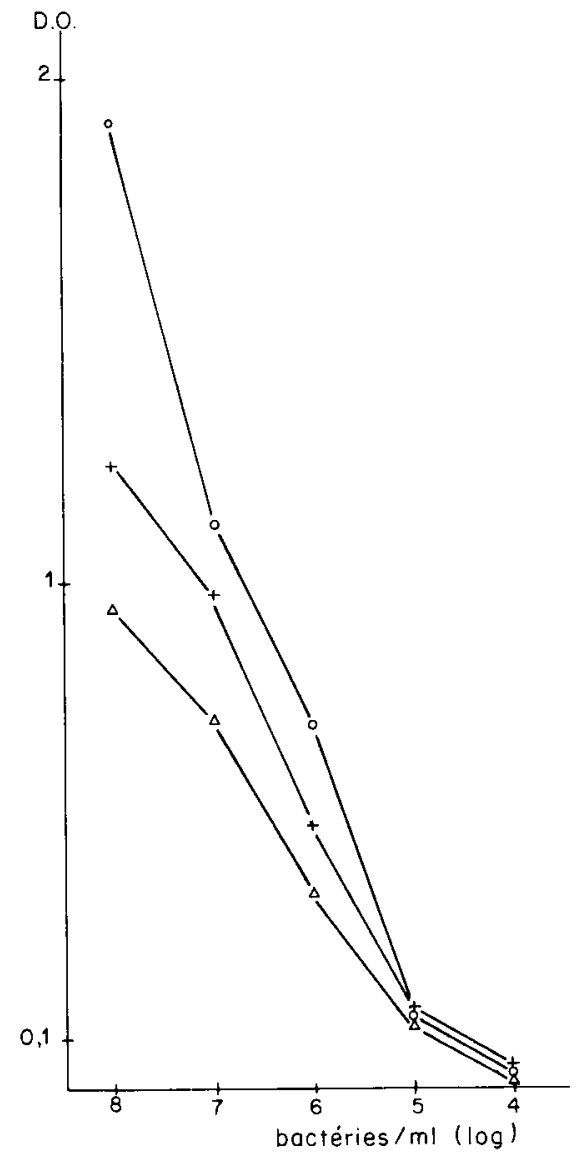

Figure 1

Comparaison de différentes immunoglobulines utilisées dans la technique ELISA appliquée à une suspension bactérienne.

Comparison between differents immunoglobulins used in ELISA technique applied to a bacterial suspension.

Les résultats sont exprimés en D.O. corrigée, à $405 \mathrm{~nm}$; temps de réaction enzymatique $l \mathrm{~h}$.

Results are expressed in corrected O.D. at $405 \mathrm{~nm}$; enzymatic reaction time: $1 \mathrm{~h}$.

○__ Immunoglobulines de lapin/Sépharose 4B-protéine A.

Rabbit immunoglobulins/Sepharose 4B-protein A.

$\triangle-\triangle$ Immunoglobulines de lapin/Sépharose $4 B$-cellules bactériennes: Ps. phaseolicola 1882.

Rabbit immunoglobulins/Sepharose 4B-bacterial cells : Ps. phaseolicola 1882.

+_ + Immunoglobulines de poulet/Sépharose 4B-cellules bactériennes: Ps. phaseolicola 1882.

Chicken immunoglobulins/Sepharose 4B-bacterial cells: Ps. phaseolicola 1882.

Conditions expérimentales.

Experimental conditions.

Revêtement : immunoglobulines lapin ou poulet $\left[10 \mu \mathrm{g} \mathrm{ml} l^{-1}\right], 5 \mathrm{~h} a ̀$ $37^{\circ} \mathrm{C}$.

Coating: rabbit or chicken immunoglobulins [10 $\left.\mu \mathrm{g} \mathrm{ml}^{-1}\right]$, $5 \mathrm{hrs,}$ $+37^{\circ} \mathrm{C}$.

Echantillon: suspension bactérienne, $18 \mathrm{~h}$ à $28^{\circ} \mathrm{C}$.

Sample: bacterial suspension, overnight, $+28^{\circ} \mathrm{C}$.

Conjugué: immunoglobulines de lapin, dilué au millième, $4 \mathrm{~h}$ à $37^{\circ} \mathrm{C}$.

Conjugate: rabbit immunoglobulins, diluted one to 1000, $4 \mathrm{hrs,}$ $+37^{\circ} \mathrm{C}$.

de lapin et de poulet obtenus à partir de la colonne Sépharose-bactéries comparativement aux immunoglobulines éluées de la colonne Sépharose-protéine A. Les valeurs sont exprimées en densité optique corrigée par rapport à des témoins de réaction sans échantillon bactérien. Dans ces conditions, en $1 \mathrm{~h}$ de révélation enzymatique, une concentration égale à $10^{6}$ cellules $/ \mathrm{ml}$ d'échantillon donne une réaction positive nette. Dans le même intervalle de temps, un début de réaction colorimétrique s'observe avec $10^{5}$ cellules $/ \mathrm{ml}$.

La sensibilité de la technique appliquée à la détection a été étudiée avec des concentrations bactériennes comprises entre $10^{7}$ et $10^{2}$ germes $/ \mathrm{ml}$. L'évolution de la réaction enzymatique a été notée dans le temps. A chacune des valeurs (moyenne de 6 répétitions) est affectée une valeur d'écart-type (tabl. 1). Dans ces conditions (écart-type et densité optique du témoin de réaction), la réaction est positive rapidement $(15 \mathrm{mn})$ jusqu'à $10^{6}$ bactéries $/ \mathrm{ml}$. En $1 \mathrm{~h}$, la réaction enzymatique est positive et intense jusqu'à $10^{6}$ bactéries $/ \mathrm{ml}$, faible mais nette jusqu'à $10^{5}$ bactéries $/ \mathrm{ml}$. La réaction positive obtenue avec $10^{5}$ bactéries $/ \mathrm{ml}$ s'intensifie dans le temps. Elle est nette au bout de $2 \mathrm{~h}$ et devient intense au-delà de $3 \mathrm{~h}$. La limite de sensibilité est représentée par la concentration de $10^{4}$ bactéries $/ \mathrm{ml}$ pour laquelle les valeurs de densité optique enregistrées sont très proches de celle des témoins de réaction (TR). Il faut attendre $4 \mathrm{~h}$ de réaction enzymatique pour obtenir une valeur de densité optique corrigée positive mais très faible. La sensibilité de la méthode, pour cette concentration, est fonction de la durée de la révélation enzymatique. La même constatation est faite pour les concentrations supérieures pour lesquelles les densités optiques corrigées augmentent dans le temps. Il en est de même pour les écarts-type, ce qui signifie que les variations des résultats d'un essai à l'autre augmentent au cours du temps de développement de la réaction enzymatique. En dessous de $10^{4}$ bactéries/ml, les valeurs de densité optique enregistrées ne sont pas significativement différentes de celles obtenues avec les témoins de réaction. Par ailleurs, le réglage du zéro de l'appareil de lecture est soumis à une variation de l'ordre de 0,02 , valeur que l'on considère comme nulle pour l'ensemble des résultats.

Il a été vérifié, en outre, que l'échantillon bactérien n'interfère pas avec l'activité de l'enzyme couplée aux immunoglobulines. Les densités optiques obtenues avec les témoins bactéries (immunoglobulines de lapin et de poulet - bactéries - tampon substrat et substrat) sont inférieures aux témoins de réaction (TR). L'évolution de ces valeurs est très faible dans le temps.

\section{B. Détection de la suspension bactérienne par la techni- que de double couche}

Dans nos conditions expérimentales, la sensibilité de détection de la suspension bactérienne n'est pas améliorée par rapport aux résultats obtenus avec la méthode "sandwich». Il faut attendre $6 \mathrm{~h}$ de réaction enzymatique pour obtenir une densité optique corrigée de 0,12 pour $10^{5}$ bactéries/ml alors qu'une valeur équivalente peut être obtenue en $60 \mathrm{mn}$ environ pour la même concentration bactérienne par la méthode «sandwich». Toutes les réactions de double couche sont réalisées en plaques et lues au spectrophotomètre. Cette technique de double couche plus longue dans la réalisation que la méthode "sandwich" à 3 étapes essentielles ne présente pas d'intérêt dans la détection de bactéries phytopathogènes. C"est pourquoi dans la suite de ce travail, il ne sera plus fait mention que de la méthode dite «sandwich».

\section{Détection des filtrats de suspension bactérienne}

Les résultats de comparaison entre le test ELISA utilisant respectivement la suspension bactérienne (SB) et les filtrats correspondants (FB) figurent au tableau 2. Les mesures de 


\section{TABLEAU 1}

Sensibilité de la technique ELISA exprimée en termes de concentration bactérienne.

La D.O. est lue à $405 \mathrm{~nm}$ : les résultats sont la moyenne de 6 valeurs avec l'écart-type.

$T R$ témoin de réaction sans échantillon (suspension bactérienne).

Sensitivity of ELISA expressed in terms of bacterial concentration.

$O . D$. at $405 \mathrm{~nm}$ : mean valuc of six measures \pm standard deviation.

$T R$ control i.e. without any sample (bacterial suspension).

\begin{tabular}{|c|c|c|c|c|c|c|c|}
\hline $\begin{array}{l}\text { Concentration bactérienne } \mathrm{ml}^{-1} \\
\text { Bacterial concentration } \mathrm{ml}^{-1} \\
\text { Temps de réaction (min) } \\
\text { Reaction time (min) }\end{array}$ & $10^{7}$ & $10^{6}$ & $10^{5}$ & $10^{4}$ & $10^{3}$ & $10^{2}$ & TR \\
\hline 15 & $0,40 \pm 0,02$ & $0,30 \pm 0,06$ & . & . & . & . & . \\
\hline 60 & $1,11 \pm 0,04$ & $0,67 \pm 0,02$ & $0,19 \pm 0,02$ & $0,11 \pm 0$ & $0,095 \pm 0,05$ & $0,09 \pm 0,01$ & $0,10 \pm 0,02$ \\
\hline 120 & 2 & $1,16 \pm 0,02$ & $0,31 \pm 0,05$ & $0,14 \pm 0$ & $0,12 \pm 0,01$ & $0,09 \pm 0,01$ & $0,10 \pm 0,02$ \\
\hline 180 & . & $1,88 \pm 0,08$ & $0,43 \pm 0,07$ & $0,16 \pm 0$ & $0,13 \pm 0,02$ & $0,11 \pm 0,02$ & $0,11 \pm 0,02$ \\
\hline 240 & . & 2 & $0,59 \pm 0,11$ & $0,18 \pm 0,02$ & $0,13 \pm 0,02$ & $0,13 \pm 0,02$ & $0,11 \pm 0,02$ \\
\hline
\end{tabular}

Conditions expérimentales.

Experimental conditions.

Revêtement : immunoglobulines de lapin $\left[10 \mu \mathrm{g} \mathrm{ml} \mathrm{m}^{-1}\right], 5 \mathrm{~h}$ à $37^{\circ} \mathrm{C}$.

Coating : rabbit immunoglobulins $\left[10 \mu \mathrm{g} \mathrm{ml} \mathbf{m}^{-1}\right], 5 \mathrm{hrs},+37^{\circ} \mathrm{C}$.

Echantillon bactérien, $18 \mathrm{~h}$ à $28^{\circ} \mathrm{C}$.

Sample, overnight, $+28^{\circ} \mathrm{C}$.

Conjugué : immunoglobulines de lapin-phosphatase, dilué au millième, $4 \mathrm{~h}$ à $37^{\circ} \mathrm{C}$.

Conjugate : rabbit immunoglobulins, diluted one to $1000,4 \mathrm{hrs},+37^{\circ} \mathrm{C}$.

TABLEAU 2

Technique ELISA appliquée aux suspensions bactériennes et aux filtrats correspoñdants $(0,22 \mu \mathrm{m})$.

ELISA technique applied to bacterial suspensions and related filtrates $(0.22 \mu \mathrm{m})$.

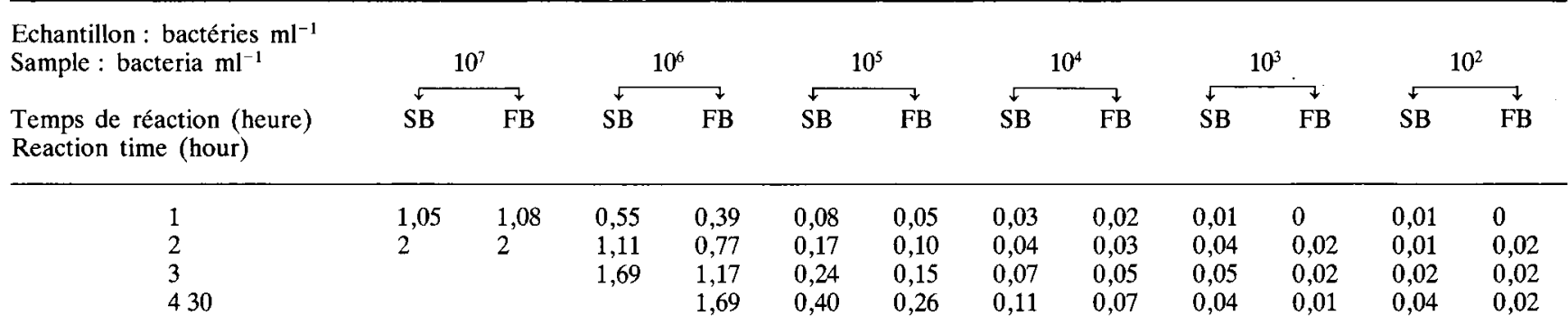

Conditions de réaction.

Experimental conditions.

Revêtement : immunoglobulines de lapin $\left[10 \mu \mathrm{g} \mathrm{ml} \mathrm{m}^{-1}\right], 5 \mathrm{~h}$ à $37^{\circ} \mathrm{C}$.

Coating: rabbit immunoglobulins $\left[10 \mu \mathrm{g} \mathrm{ml} \mathrm{m}^{-1}\right], 5 \mathrm{hrs},+37^{\circ} \mathrm{C}$.

Echantillon : $18 \mathrm{~h}$ à $28^{\circ} \mathrm{C}$.

Sample : overnight, $+28^{\circ} \mathrm{C}$.

Conjugué : immunoglobulines de lapin-phosphatase, dilué au millième, $4 \mathrm{~h}$ à $37^{\circ} \mathrm{C}$.

Conjugate : rabbit immunoglobulins-phosphatase, diluted one to $1000,4 \mathrm{hrs},+37^{\circ} \mathrm{C}$.

SB : Suspension bactérienne. Bacterial suspension.

FB : Filtrat. Filtrate.

Les résultats sont exprimés en D.O. corrigée à $405 \mathrm{~nm}$. Results are expressed in corrected O.D. at $405 \mathrm{~nm}$.

densité optique sont faites dans le temps et sont exprimées en densité optique corrigée. Dans les limites de l'écart-type (tabl. 1), les résultats du tableau 2 confirment ceux du tableau 1. Les valeurs de densité optique corrigée sont nettes et apparaissent lentement pour une concentration bactérienne de $10^{5}$ bactéries $/ \mathrm{ml}$ et le filtrat de culture correspondant. La limite de sensibilité est représentée par la concentration $10^{4}$ bactéries $/ \mathrm{ml}$ et le filtrat correspondant pour laquelle la réaction positive est faible et très lente à apparaître. Il faut remarquer par ailleurs que les valeurs de densité optique corrigée sont toujours plus faibles pour le filtrat (FB) que pour la suspension bactérienne (SB) correspondante.

\section{Etude avec les culots et filtrats bactériens}

Les différents culots bactériens $(\mathrm{Ci})$ sont remis en suspension dans le liquide physiologique et ajustés à une concen- 
tration de $10^{8}$ bactéries/ml environ. Les surnageants ( $\mathrm{Si}$ ) après filtration sur membrane de $0,22 \mu \mathrm{m}$ sont dosés au spectrophotomètre à $280 \mathrm{~nm}$. L'ensemble des échantillons $\mathrm{Ci}$ et $\mathrm{Si}$ est testé en ElISA (tabl.3). Quel que soit l'échantillon considéré, suspension obtenue à partir des différents culots ou liquides filtrés, la réaction ELISA en $60 \mathrm{mn}$ est intense. La plus faible valeur de réaction obtenue avec le filtrat 1 est due à sa trop forte concentration moléculaire qui ne correspond pas à la zone d'équivalence (excès d'antigène). Les autres filtrats sont moins concentrés

\section{TABLEAU 3}

La technique ELISA appliquée aux suspensions bactériennes et aux filtrats correspondants après lavages par centrifugation. ELISA technique applied to bacterial suspensions and corresponding filtrates after serial washings by centrifugation.

\begin{tabular}{lccccccccc}
\hline \hline & $\begin{array}{l}\text { Lavages } \\
\text { washing }\end{array}$ & 1 & 2 & 3 & 4 & 5 & 6 & 7 & 8 \\
$\begin{array}{l}\text { Echantillon } \\
\text { Sample }\end{array}$ & & & & & & & & & \\
\hline $\begin{array}{l}\text { Culot (Ci) } \\
\text { Sediment }\end{array}$ & 1,11 & 1,12 & 1,11 & 1,11 & 1,11 & 1,16 & 1,21 & 1,17 & 1,16 \\
$\begin{array}{l}\text { Filtrat } \\
\text { Filtrate }\end{array}$ & 0,66 & 0,84 & 0,89 & 0,86 & 0,94 & 0,96 & 0,97 & 0,95 & 0,80 \\
\hline
\end{tabular}

Conditions expérimentales.

Experimental conditions.

Revêtement: immunoglobulines de lapin $\left[10 \mu \mathrm{g} \mathrm{ml}{ }^{-1}\right], 5 \mathrm{~h}$ à $37^{\circ} \mathrm{C}$.

Coating : rabbit immunoglobulins $\left[10 \mu \mathrm{g} \mathrm{ml^{-1 }}\right.$, $5 \mathrm{hrs}$, $\Rightarrow 37^{\circ} \mathrm{C}$.

Echantillon: (Ci ou Si), $18 \mathrm{~h}$ à $28^{\circ} \mathrm{C}$.

Sample ( $\mathrm{Ci}$ or $\mathrm{Si}$ ), overnight, $+28^{\circ} \mathrm{C}$.

Conjugué : immunoglobulines de lapin-phosphatase, dilué au millième, $4 \mathrm{~h}$ à $37^{\circ} \mathrm{C}$.

Conjugate : rabbit immunoglobulins-phosphatase, diluted one to 1000,4 hrs $+37^{\circ} \mathrm{C}$.

Révélation enzymatique : $1 \mathrm{~h}$.

Reading time after substrate application: $1 \mathrm{~h}$.

Les résultats sont exprimés en densité optique corrigée. Chaque culot bactérien (Ci) est remis en suspension et ajusté à 107 bactéries/ml. Results are expressed in terms of corrected O.D. Bacterial sediments (Ci) are resuspended and adjusted to $10^{7}$ bacteria/ml.

comme le montrent les mesures spectrophotométriques à $280 \mathrm{~nm}$ (tabl. 4). Il faut remarquer que, même après 9 lavages, la densité optique à $280 \mathrm{~nm}$ n'est pas encore annulée.

\section{TABLEAU 4}

Densité optique à $280 \mathrm{~nm}$ des différents surnageants de lavages. Optical density at $280 \mathrm{~nm}$ of supernatants corresponding with serial washings.

\begin{tabular}{lcccccccccc}
\hline \hline Surnageant de lavage & 1 & 2 & 3 & 4 & 5 & 6 & 7 & 8 & 9 \\
\hline Supernatants & $>1,0$ & 0,06 & & \multicolumn{3}{c}{$0,05 \pm 0,01$} \\
\hline
\end{tabular}

\section{E. Sensibilité de la technique dans la détection du filtrat bactérien}

Des dilutions ont été faites à partir d'un filtrat $\mathrm{S} 1$ dont la concentration est déterminée d'après la densité optique d'une solution de sérum albumine de référence, à $280 \mathrm{~nm}$ (D.O. $280 \mathrm{~nm}=0,5 \mathrm{mg} / \mathrm{ml}$ ). Dans les conditions expérimentales, une concentration de filtrat de l'ordre de $10 \mathrm{ng}$ est détectée en $60 \mathrm{mn}$ de révélation enzymatique.

\section{F. Détection de Ps. phaseolicola dans les extraits de feuilles inoculées de haricot}

Un même poids de feuilles de haricot présentant, soit des taches graisseuses dans le cas de la réaction de maladie, soit des nécroses d'hypersensibilité, est broyé dans un même volume de tampon. Les résultats sont exprimés en densité optique corrigée par rapport à la densité optique d'un extrait de feuilles saines (tabl. 5).

On note dans ce tableau une réaction positive intense, rapide, due à Ps. phaseolicola $\mathrm{n}^{\circ} 1882$ pathogène du haricot. La valeur de 0,02 obtenue avec l'extrait contaminé par Ps. syringae $\mathrm{n}^{\circ} 1779$ filtré, après $270 \mathrm{mn}$ de réaction enzymatique ne peut être considérée comme positive compte tenu de la remarque faite précédemment.

L'extrait contaminé par Ps. syringae $\mathrm{n}^{\circ} 1769$ donne un résultat positif faible et lent à apparaître ; l'extrait contaminé par Ps. syringae no 1447 donne également une réaction positive en $270 \mathrm{mn}$. Ces résultats positifs, confirmés à l'examen par immunofluorescence sont dus aux réactions sérologiques croisées des souches Ps. syringae $\mathrm{n}^{\circ} 1769$ et $\mathrm{n}^{\circ} 1147$ dans l'anti-sérum Ps. phaseolicola $\mathrm{n}^{\circ} 1882$. Il est intéressant de noter que le test ELISA, sans supprimer l'existence de réactions sérologiques croisées: permet dans le cas d'une lecture rapide d'obtenir une réponse spécifique.

\section{G. Détection de PS. phaseolicola dans les macérats de graines}

Le liquide de macération d'un même poids de graines de haricot est testé à l'état brut ou après filtration (tabl. 6). En $1 \mathrm{~h}$ de réaction, 5 lots paraissent positifs : «Vernel » (lot $\mathrm{A}$ et lot D), «Amilcar», «Opal» et «Arly ». Ces lots sont également trouvés positifs en immunofluorescence. Une différence d'intensité colorimétrique apparaît avec la variété «Amilcar » entre les macérats filtrés et non filtrés. Il semble que cette différence soit propre au lot puisque, dans toutes les autres réactions positives, les résultats obtenus 
TABLEAU 5

La technique ELISA appliquée aux extraits de feuilles contaminées de haricot et aux filtrats correspondants $(0,22 \mu \mathrm{m})$. ELISA technique applied to contaminated bean leaf extracts and to corresponding filtrates $(0.22 \mu \mathrm{m})$.

Temps de réaction $(\mathrm{mn})$

Reaction time (mn)

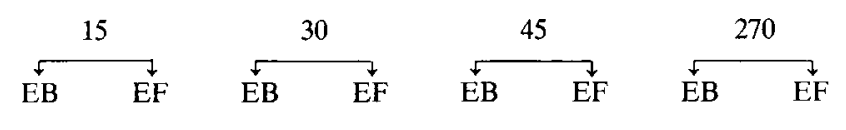

Immunofluorescence

Bactéries

Bacteria

\begin{tabular}{|c|c|c|c|c|c|c|c|c|c|}
\hline $\begin{array}{l}\text { Pseudomonas phaseolicola } \mathrm{n}^{\circ} 1882 \\
\text { (Phaseolus vulgaris) }\end{array}$ & 0,13 & 0,1 & 0,27 & 0,24 & 0,42 & 0,41 & 2 & 2 & + \\
\hline $\begin{array}{l}\text { Pseudomonas phaseolicola } \mathrm{n}^{\circ} 1659 \\
\text { (Phaseolus aureus) }\end{array}$ & 0 & 0 & 0 & 0 & 0 & 0 & 0 & 0 & - \\
\hline $\begin{array}{l}\text { Pseudomonas syringae } \mathrm{n}^{\circ} 1779 \\
\text { (Citrus sinensis) }\end{array}$ & 0 & 0 & 0 & 0 & 0 & 0 & 0 & 0,02 & - \\
\hline $\begin{array}{l}\text { Pseudomonas syringae } \mathrm{n}^{\circ} 1769 \\
\text { (Pisum sativum) }\end{array}$ & 0 & 0 & 0 & 0 & 0 & 0 & 0,10 & 0,07 & $(+)$ \\
\hline $\begin{array}{l}\text { Pseudomonas syringae } \mathrm{n}^{\circ} 1147 \\
\text { (Pyrus communis) }\end{array}$ & 0 & 0 & 0 & 0 & 0 & 0 & 0,20 & 0,15 & + \\
\hline
\end{tabular}

Conditions expérimentales. Experimental conditions.

Revêtement : immunoglobulines de lapin $\left[10 \mu \mathrm{g} \mathrm{ml} l^{-1}\right], 18 \mathrm{~h}$ à $4^{\circ} \mathrm{C}$.

Coating : rabbit immunoglobulins $\left[10 \mu \mathrm{g} \mathrm{ml}{ }^{-1}\right]$, overnight, $+4{ }^{\circ} \mathrm{C}$.

Echantillon : extrait de feuilles, $2 \mathrm{~h}$ à $28^{\circ} \mathrm{C}$.

Sample : leaf extract, $2 \mathrm{hrs},+28^{\circ} \mathrm{C}$.

Conjugué : immunoglobulines de lapin-phosphatase, dilué au huit centième, $4 \mathrm{~h}$ à $37^{\circ} \mathrm{C}$.

Conjugate : rabbit immunoglobulins-phosphatase, diluted one to $800,4 \mathrm{hrs},+37^{\circ} \mathrm{C}$.

Les résultats sont exprimés en D.O. corrigée, à $405 \mathrm{~nm}$.

Immunofluorescence : anti-sérum Ps. phaseolicola 1882 dilué au millième : + réaction positive nette $;(+)$ réaction positive faible ; - pas de réaction.

Results are expressed in corrected O.D., at $405 \mathrm{~nm}$.

Immunofluorescence: antiserum Ps. phaseolicola 1882, diluted one to 1000 : + positive strong reaction; $(+)$ positive, weak reaction ; - negative.

avec les filtrats sont parallèles à ceux obtenus avec extraits bruts. Les densités optiques égales à 0,01 ou 0,02 soit au bout de $30 \mathrm{mn}$ de réaction ("Vernel », lot B et lot C), soit après 60 ou $120 \mathrm{mn}$ («Calvy », «Farcy») ne peuvent pas être considérées comme positives.

\section{DISCUSSION}

Les résultats obtenus dans la détection bactérienne en culture pure ou dans les extraits végétaux confirment les travaux déjà présentés sur la technique ELISA en phytobactériologie. Mais de plus, ce travail montre que la détection de $P$ s. phaseolicola peut se faire aussi bien à partir de filtrats de la suspension bactérienne provenant soit de culture pure soit d'échantillons végétaux naturellement contaminés.

Le test d'immunofluorescence pratiqué sur les parois des cupules a permis de mettre en évidence la présence de nombreuses bactéries fluorescentes. Il semble bien que les corps bactériens soient fixés par la couche d'anticorps homologues qui tapissent les parois du support.

Les réactions positives enregistrées avec les filtrats de culture bactérienne ou d'extraits végétaux montrent que les anticorps homologues fixent également les métabolites bactériens. Ces métabolites sont excrétés directement dans le milieu ou transitent plus ou moins longtemps à travers la paroi bactérienne. Ces molécules, soit à l'état libre dans le filtrat soit à l'état de transit dans la paroi bactérienne, sont à l'origine des réactions positives selon le test ELISA. Il est probable que des antigènes de structure constitutifs de la paroi et non excrétés dans le milieu soient aussi à l'origine de réactions positives enregistrées avec les suspensions bactériennes. Ces considérations montrent qu'il est incorrect d'exprimer la sensibilité du test ELISA appliqué à la détection de bactéries phytopathogènes en terme de corps bactériens par ml d'échantillon. Il nous apparaît plus correct de l'exprimer en terme de quantité d'antigènes décelables, solubles ou non. La valeur approximative de $10 \mathrm{ng}$ de protéines par $\mathrm{ml}$ de filtrat, par référence à l'albumine bovine détectée en ELISA, correspond aux sensibilités données en général pour cette technique.

Compte tenu de ces remarques, il semble que la technique ELISA soit parfaitement bien adaptée à la détection de la bactérie par l'intermédiaire de ses ou d'un métabolite(s) spécifique(s) présents dans les liquides d'extraction et de macération de végétaux contaminés. Dans ces conditions, elle se ramène à son application première qui est la détection de molécules et non de cellules bactériennes.

Les tests de détection pourront être pratiqués sur ces liquides d'extraction et de macération par la mise en évidence, soit des corps bactériens par immunofluorescence, soit du complexe cellules-métabolites par le test ELISA. Cependant, ce dernier ne peut servir à quantifier une population bactérienne que s'il existe, dans des conditions définies, une corrélation étroite entre la quantité de métabolites produits et une concentration de bactéries.

La spécificité de la réaction ELISA se dégage des essais réalisés avec les extraits de feuilles contaminées. La réaction spécifique dans le cas du système homologue Ps. phaseolicola-sérum anti Ps. phaseolicola est rapide et intense. Avec les systèmes hétérologues $P s$. syringae $n^{\circ} 1769$ et $n^{\circ} 1147$, la réaction est lente à apparaître et de bien moindre intensité en $270 \mathrm{mn}$ de réaction surtout avec Ps. syringae $\mathrm{n}^{0} 1769$. Dans le temps, la spécificité de la réaction ELISA est la 
TABLEAU 6

La technique ELISA appliquée aux macérats de graines contaminées de haricot et aux filtrats correspondants $(0,22 \mu \mathrm{m})$. ELISA technique applied to macerating liquids of contaminated bean seeds and to corresponding filtrates $(0.22 \mu \mathrm{m})$.

\begin{tabular}{|c|c|c|c|c|c|c|c|}
\hline \multirow{2}{*}{$\begin{array}{l}\text { Temps de réaction }(\mathrm{mn}) \\
\text { Reaction time }(\mathrm{mn}) \\
\text { Echantillons } \\
\text { Sample }\end{array}$} & \multicolumn{2}{|c|}{30} & \multicolumn{2}{|c|}{60} & \multicolumn{2}{|c|}{120} & \multirow{2}{*}{ Immunofluorescence } \\
\hline & $\stackrel{\downarrow}{\text { EB }}$ & $\stackrel{\downarrow}{\mathrm{EF}}$ & $\stackrel{\downarrow}{\mathrm{EB}}$ & $\mathrm{EF}$ & $\stackrel{\downarrow}{\mathrm{EB}}$ & EF & \\
\hline Vernel $(\operatorname{lot} A)$ & 0,18 & 0,16 & 0,36 & 0,32 & 0,81 & 0.75 & + \\
\hline Vernel (lot B) & 0,01 & 0 & 0 & 0 & 0,01 & 0 & - \\
\hline Vernel (lot C) & 0,02 & 0 & 0 & 0 & 0 & 0 & - \\
\hline Hanfa & 0 & 0 & 0 & 0 & 0 & 0 & - \\
\hline Amilcar & 0,28 & 0,14 & 0,67 & 0,27 & 1,21 & 0,69 & + \\
\hline Calvy & 0 & 0 & 0,01 & 0,01 & 0,02 & 0,02 & - \\
\hline Farcy & 0 & 0 & 0 & 0 & 0 & 0,02 & - \\
\hline Opal & 0,10 & 0,07 & 0,17 & 0,14 & . & . & + \\
\hline Arly & 0,23 & 0,19 & 0,44 & 0,35 & . & . & + \\
\hline Vernel (lot D) & 0,14 & 0,11 & 0,33 & 0,21 & . & . & + \\
\hline
\end{tabular}

Conditions expérimentales.

Experimental conditions.

Revêtement : immunoglobulines de lapin $\left[10 \mu \mathrm{g} \mathrm{ml}^{-1}\right], 18 \mathrm{~h}$ à $4^{\circ} \mathrm{C}$.

Coating : rabbit immunoglobulins $\left[10 \mu \mathrm{g} \mathrm{m}^{-1}\right]$, overnight, $+4{ }^{\circ} \mathrm{C}$.

Echantillon : $2 \mathrm{~h}$ à $28^{\circ} \mathrm{C}$.

Sample : 2 hrs, $+28^{\circ} \mathrm{C}$.

Conjugué : immunoglobulines de lapin-phosphatase, $4 \mathrm{~h}$ dilué au huit centième, $4 \mathrm{~h}$ à $37^{\circ} \mathrm{C}$.

Conjugate : rabbit immunoglobulins-phosphatase, diluted one to $800,4 \mathrm{hrs},+37^{\circ} \mathrm{C}$.

$\therefore$ pas de lecture.

. : no reading.

Les résultats sont exprimés en D.O. corrigée, à $405 \mathrm{~nm}$.

Immunofluorescence : anti-sérum $P$ s. phaseolicola, absorbé.

Results are expressed in corrected O.D. at $405 \mathrm{~nm}$.

Immunofluorescence absorbed anti-serum Ps. phaseolicola.

EB : liquide de macération. Macerating liquid.

EF : filtrat. Filtrate.

. : non testé. Not recorded.

même qu'en immunofluorescence. Mais, en ELISA, si Ps. phaseolicola est présent à forte concentration, la réaction lue est intense, rapide et spécifique. Par contre, si Ps. phaseolicola est peu concentré dans l'échantillon, de l'ordre de $10^{4}$ bactéries $/ \mathrm{ml}$, il est difficile de distinguer la réaction homologue lente et faible de la réaction hétérologue ; on peut en outre supposer des concentrations importantes de bactéries hétérologues, ce qui pourrait donner des réactions croisées intenses et rapides. Avec les extraits contaminés par Ps. syringae $\mathrm{n}^{\text {os }} 1147$ et 1769 , les concentrations bactériennes des souches hétérologues sont faibles ou les sites antigéniques communs avec Ps. phaseolicola sont peu nombreux. Seule, l'utilisation d'antisérums spécifiques du germe à détecter permettra l'obtention de résultats spécifiques.

Une voie de recherche intéressante nous apparaît. Elle consiste en l'extraction et la purification de métabolites spécifiques de la bactérie. L'utilisation d'antisérums préparés à partir de tels antigènes solubles devrait permettre la détection de très faibles quantités de ces métabolites spécifiques dans les milieux naturels contaminés. Une telle méthodologie serait un apport précieux dans l'étude précise des relations hôtes-bactéries in vivo.

La reproductibilité des résultats au sein d'un même laboratoire ou entre différents laboratoires exige la standardisation des réactifs utilisés notamment du conjugué anticorps-enzyme. La méthode ELISA « sandwich » suppose la préparation d'un réactif spécifique à chaque germe ou molécule type recherché donc la nécessité d'une gamme éventuelle de conjugués pour les besoins d'un laboratoire de détection. L'utilisation d'un conjugué dit universel employé dans la technique de double couche résoudrait cet inconvénient mais, dans nos conditions expérimentales, cette méthode n'a pas amélioré le résultat. La standardisation devra porter sur le schéma d'immunisation: obtention de sérums d'animaux hyperimmunisés avec la présence d'anticorps à haute affinité (AvRAMEAS et al., 1978), la préparation du conjugué anticorps-enzyme proprement dite. Ce travail a été réalisé avec la phosphatase d'Escherichia coli, ce qui permettait l'utilisation d'un tampon substrat approprié à l'optimum d'activité de cet enzyme. Les travaux traitant ELISA en bactériologie végétale indiquent par contre, pour la plupart, l'utilisation de la phosphatase alcaline (type VII) d'intestin de veau avec la diéthanolamine comme tampon substrat. L'utilisation du tampon tris$\mathrm{HCl}, \mathrm{NaCl}$ nous a permis d'éviter les colorations non spécifiques souvent constatées avec la diéthanolamine. Avec l'emploi d'une phosphatase alcaline, plus active commercialement que la phosphatase d'E. coli, et un tampon convenable pour la révélation enzymatique on peut espérer une amélioration de la sensibilité des résultats. La qualité du couplage constitue un autre paramètre important capable d'agir sur la sensibilité de la technique et sur la qualité des résultats. La présence d'un nombre plus ou 
moins élevé de molécules d'enzymes libres dans le mélange contribue à augmenter les bruits de fond ou réactions non spécifiques. La séparation des molécules non couplées (anticorps et enzymes) des molécules couplées (AVRAMEAS et al., 1978) est sans nul doute à préconiser ainsi qu'une standardisation pour la détermination de la dilution du conjugué à utiliser.
Reçu le 18 septembre 1981. Accepté le 8 décembre 1981.

\section{RÉFÉRENCES BIBLIOGRAPHIQUES}

Avrameas S., Guilbert B., 1971. Dosage enzymo-immunologique de protéines à l'aide d'immunoadsorbants et d'antigènes marqués aux enzymes C.R. Acad. Sci., Paris Sér. D, 273, 2705-2707.

Avrameas S., Ternynck T., Guesdon J. L., 1976. Some general remarks about immunoenzymatic techniques. First Intern. Sympos. Immunoenz. Tech., INSERM. sympos., 2, 1-6.

Avrameas S., Ternynck T., Guesdon J. L., 1978. Coupling of enzymes to antibodies and antigens. Scand. J. Immunol., 8, suppl. 7, 7-23.

Avrameas S., Uriel J., 1966. Méthode de marquage d'antigènes et d'anticorps avec des enzymes et son application en immunodiffusion. C.R. Acad. Sci., Paris Sér. D, 262, 2543-2545.

Barzic M. R., 1980. Méthode d'obtention d'un conjugué entre des bactéries phytopathogènes et un support polysaccharidique. C.R. Acad. Sci., Paris, 291, sér. D, 589-592.

Berger J. A., May S. N., Berger L. R., Bohlool B. B., 1979. Colorimetric enzyme linked immunosorbent assay for the identification of strains of Rhizobium in culture and in the nodules of lentils. Appl. environ. Microbiol., 642-646.

Cambra M., Lopez M. M., 1978. Titration of Agrobacterium radiobacter var. tumefaciens antibodies by using enzyme labelled anti rabbit globulins (ELISA indirect method). Proc. 4th Int. Conf. Plant Path. Bact., Angers, 327-331.

Caron M., Copeman R. J., 1981. Factors affecting detection of Erwinia carotovora var. atroseptica by ElISA. Phytopathology, 71, 2.

Claflin L. E., Uyemoto J. K., 1978. Serodiagnosis of Corynebacterium sepedonicum by enzyme linked immunosorbent assay. Phytopathol. News, 12.

Clark M. F., Adams A. N., 1977. Characteristics of the microplate method of enzyme-linked immunosorbent assay for the detection of plant viruses. J. gen. Virol., 34, 475-483.

Coléno A., 1968. Utilisation de la technique d'immunofluorescence pour le dépistage de Pseudomonas phaseolicola (Burkh.) Dowson dans les lots de semences. C.R. Acad. Agric. Fr., 54 (12), 10161020 .
Coons A. H., Creech H. J., Jones R. N., 1941. Immunological properties of an antibody containing a fluorescent group. Proc. Soc. exp. Biol. Med., 47, 200-202.

Engvall E., Perlmann P., 1971. Enzyme linked immunosorbent assay. Quantitative assay of immunoglobulin G. Immunochemistry, 8, 871-874.

Garen A., Levinthal C., 1960. A fine structure genetic and chemical study of the enzyme alkaline phosphatase of E. coli. I. Purification and characterization of alkaline phosphatase. Biochim. biophys. Acta, 38, 470-483.

Nakane P. K., Sri Ram., Pierce G. B., 1966. Enzyme labelled antibodies for light and electron microscopic localisation of antigens. J. Histochem. Cytochem., 14, 789-791.

Scherrer R., Bernard S., 1977. Application d'une technique immunoenzymatique. (ELISA) à la détection d'un rotavirus bovin et des anticorps dirigés contre lui. Ann. Microbiol. (Inst. Pasteur), 128A, 499-510.

Stevens W. A., Tsiantos J., 1979. The use of enzyme linked immunosorbent assay (ELISA) for the detection of Corynebacterium michiganense in tomatoes. Microbios lett., 10, 29-32.

Trigalet A., Samson R., Coléno A., 1978. Problems related to the use of serology in phytobacteriology. Proc. 4th Int. Conf. Plant Path. Bact., Angers, 271-288.

Trigalet A., Samson R., Digat B., Lemattre M., 1979. Détection des bactérioses transmises par les semences. $16^{\mathrm{e}}$ Coll. Soc. fr. Phytopathol. Ann. Phytopathol., 11 (4), 543-544.

Van Veemen B. K., Schurrs A. H. W. M., 1971. Immunoassay using antigen enzyme conjugates. FEBS lett., 15, 232-236.

Voller A., Bartlett A., Bidwell D. E., Clark M. F., Adams A. N., 1976. The detection of viruses by enzyme linked immunosorbent assay. J. gen. Virol., 33, 165-167.

Vrugging H., 1978. Enzyme linked immunosorbent assay in the serodiagnosis of plant pathogenic bacteria. Proc. 4th Int. Conf. Plant Path. Bact., Angers, 307-310.

Weaver W. M., Guthrie J. W., 1978. Enzyme linked immunospecific assay application to the detection of seed borne bacteria. Phytopathol. News, 12, 156. 